\title{
Afrontamiento al Estrés: Adaptación del Cuestionario COPE en Universitarios de Lima
}

\author{
Stress Coping: Adaptation of the Questionnaire COPE in University's Students of \\ Lima
}

\author{
Mónica Cassaretto Bardales ${ }^{1}$ y Cecilia Chau Perez-Aranibar ${ }^{2}$
}

\begin{abstract}
Resumen
Se analizan las propiedades psicométricas del inventario de estimación del afrontamiento COPE de 60 ítems (Carver, Scheier, \& Weintraub, 1989) al aplicarse en un grupo de 300 estudiantes universitarios de Lima, Perú. Los participantes tenían entre 16 y 25 años ( $M=18.27$ y D.E.=1.39 años). Los resultados finales arrojan evidencia sólida de la organización de 15 estrategias. El análisis factorial exploratorio muestra a nivel de ítems la existencia de 13 factores que explican $65.15 \%$ de varianza; las escalas afrontamiento activo y planificación por un lado y buscar soporte social por motivos instrumental y buscar soporte social por motivos emocionales aparecen fusionadas. Los índices alfa de Cronbach de las escalas oscilan entre .53 a .91. Sin embargo, análisis factoriales exploratorios y confirmatorios cuestionan la posibilidad de la organización de los estilos y encuentra débil evidencia para una estructura de 3 factores que organicen dichas escalas (afrontamiento centrado en tarea/aproximación, lo social/emocional y en la evitación).
\end{abstract}

Palabras clave: COPE, afrontamiento, estilos de afrontamiento, estrategias de afrontamiento, estudiantes universitarios

\begin{abstract}
This study analyzes the psychometric properties of the coping estimation inventory COPE of 60 items on a group of 300 university students from Lima, Peru. There ages were between 16 to 25 years old $(X=18.27$ and $S . D .=1.39$ years). The results shown strong evidence related to the 15 -strategy organization. The exploratory factorial analysis shown that 13 factors, explain $65.15 \%$ of variance. Items from Active Coping scale and the Planning scales load together on one factor as in Seeking Social Support for Emotional Reasons and Seeking Social Support for Instrumental Reasons load as one distinct factor. The internal consistency analysis for scales shows the Cronbach alpha coefficients between .53 and .91. Exploratory and confirmatory factorial question the possibility of the organization of coping styles and finds weak evidence for a structure of 3 factors (Task/Approach-oriented coping, Social/Emotional-oriented coping and Avoidance-oriented coping).
\end{abstract}

Keywords: COPE, coping, coping styles, coping strategies, university students

\footnotetext{
${ }^{1}$ Magister en Psicología Clínica de la Salud. Pontificia Universidad Católica del Perú. Av. Universitaria 1801, San Miguel, Lima 32, Perú. 511-6262000 anexo 4598. E-mail: mcassar@ pucp.edu.pe

${ }^{2}$ PhD en Psicología. Pontificia Universidad Católica del Perú. Av. Universitaria 1801, San Miguel, Lima 32, Perú. 511 6262000 anexo 4563. E-mail: cchau@ pucp.edu.pe
} 


\section{Introducción}

El Inventario Multidimensional de Estimación del Afrontamiento COPE es un instrumento creado para evaluar las diferentes maneras en que las personas responden al estrés (Carver, Scheier, $\&$ Weintraub, 1989). Actualmente es considerado una de las principales herramientas para medir tales estrategias e identificar los estilos de afrontamiento (Clark, Bormann, Cropanzano, \& James, 1995; Ficková, 2005; Folkman \& Moskowitz, 2004; Kallasmaa \& Pulver, 2000; Lyne \& Roger, 2000; Schwarzer \& Schwarzer, 1996).

El COPE tiene dos versiones, la versión situacional orientada a obtener respuestas en situaciones específicas o temporales y la versión disposicional, dirigida a evaluar respuestas típicas del sujeto. Los ítems son los mismos para ambas formas, variando solamente las instrucciones para su aplicación (Carver et al, 1989). Algunos investigadores plantean que si bien puede haber beneficios en la evaluación diferenciada de ambas versiones, consideran que ambos enfoques no son del todo discordantes. Al respecto señalan que el afrontamiento puede ser entendido tanto en términos estables (disposicionales) como temporales (situacionales), ya que dependiendo del contexto el sujeto recurrirá preferentemente a algunos de ellos (Carver et al, 1989; FernándezAbascal, 1997; Krohne 1996).

El COPE es una prueba que tiene distintas versiones. La original es la versión de 52 ítems que se organizan alrededor de 13 escalas. Estas representan las estrategias específicas de afrontamiento que usan las personas en situaciones de estrés y son: afrontamiento activo, planificación, supresión de actividades en competencia, restricción del afrontamiento, búsqueda de apoyo social por razones instrumentales, búsqueda de apoyo social por razones emocionales, reinterpretación positiva y crecimiento, aceptación, acudir a la religión, enfocar y liberar emociones, negación, desentendimiento conductual y desentendimiento mental.

El primer estudio realizado con esta versión arroja algunos resultados positivos en términos de la validez y confiabilidad; no obstante, los autores de la prueba decidieron adicionar al inventario una escala con un solo ítem: uso de alcohol y drogas. Así queda una segunda versión del COPE conformado por 14 escalas y 53 ítems (Carver et al., 1989).

Desde entonces, el inventario ha sido ampliamente utilizado en diferentes poblaciones y en diversos contextos dentro del Perú, tanto en su versión de 52 ítems como en la de 53 (Cassaretto, 2011; Cassaretto, Chau, Oblitas, \& Valdez, 2003; Cassaretto \& Paredes, 2006; Chau 1999, 2004; Chau, Morales, \& Wetzell, 2002; Gastelumendi \& Ore, 2013; Levano, 2003; Rojas, 1997; Solano, 2013). Sin embargo, pese a su considerable empleo, pocos estudios se han centrado en evaluar sus propiedades psicométricas y estructura interna, y los que lo han hecho se han restringido a analizar el funcionamiento de la versión disposicional de la prueba. Estos estudios suelen reportar buen funcionamiento de la mayoría de ítems y escalas pero también algunas dificultades en términos de consistencia interna y reproducción de la estructura factorial de segundo orden (Cassaretto, 2009; Casuso, 1996; Chau, 1999; 2004).

\section{Investigaciones en distintos países}

De los pocos estudios psicométricos a nivel mundial destaca el propio estudio de Carver et al. (1989), quienes reportan los resultados del instrumento en términos de consistencia interna y validez al ser evaluado en 978 estudiantes universitarios en Estados Unidos. Respecto a la consistencia interna de las escalas presentaron índices alfa de Cronbach entre 0.45 hasta 0.92; siendo el área débil desentendimiento mental con una alfa menor de $0.60(\alpha=0.45)$; asimismo, las mediciones test-retest dieron rangos entre $0.46 \mathrm{y}$ 0.86 a las 8 semanas.

Por otro lado, la validez de constructo fue calculada mediante un análisis factorial usando una rotación oblicua; así, se obtiene una fórmula final de 11 factores con valores eigen mayores a 1.0. Se señala que las escalas afrontamiento activo y planificación conforman un solo factor; mientras que las dos escalas de soporte conforman un segundo. Sin embargo, los autores optaron por mantener cada escala por separado por motivos teóricos. 
En un análisis factorial exploratorio de segundo orden de las escalas del COPE, Carver y sus colaboradores obtienen 4 factores que se organizan de la siguiente manera: afrontamiento orientado a la tarea (incorpora afrontamiento activo, planificación y supresión de actividades competentes), a la emoción (búsqueda de apoyo social por razones instrumentales, búsqueda de apoyo social por razones emocionales y, enfocar y liberar de emociones), a la evitación (negación, desentendimiento mental y desentendimiento conductual) y un afrontamiento más cognitivo (aceptación, restricción del afrontamiento y reinterpretación positiva). Además se reporta que la escala acudir a la religión no logra ubicarse dentro de ninguno de esos factores ya que presenta una carga factorial baja (0.23) sobre el último factor. Desafortunadamente los autores del estudio dieron poca información adicional sobre los procedimientos utilizados para este análisis.

Por otro lado, en Estados Unidos Lyne y Roger (2000) intentaron replicar el estudio de Carver y colaboradores (1989) en una investigación con 587 sujetos adultos. Estos autores utilizaron como técnica de extracción de factores el método Kaiser-Guttman. Sus resultados no pudieron replicar completamente los 13 factores o escalas originalmente propuestos. Además, en un análisis de segundo orden encontraron una estructura trifactorial para 37 ítems del COPE, los factores hallados fueron nombrados afrontamiento racional, afrontamiento emocional y afrontamiento evitativo.

Bajo este contexto, años después de su publicación, los creadores del inventario deciden incorporar cambios finales a la prueba. Añadieron 3 ítems para la escala de uso de alcohol/drogas y 4 ítems para una nueva escala llamada uso del humor, dando paso así a una tercera versión del COPE conformada por 60 ítems y 15 escalas o estrategias de afrontamiento (como se cita en Kallasmaa \& Pulver, 2000). Esta nueva reorganización del COPE trae consigo indefectiblemente una serie nueva de estudios acerca de sus propiedades psicométricas, los cuales han respaldado, en su mayoría, el funcionamiento de las estrategias de afrontamiento planteadas, aunque aún existe cierta controversia respecto al número de factores que conforman los estilos.

Uno de los primeros estudios en analizar esta última versión fue el llevado a cabo en Reino Unido por Ingledew, Hardy, Cooper y Jemal (2013), quienes realizaron una investigación que buscaba establecer una relación entre el afrontamiento con las conductas de salud en 256 adultos. Estos autores hallan índices de consistencia interna para las estrategias entre 0.37 y 0.91 , siendo nuevamente la escala con consistencia más baja desentendimiento mental al presentar un alfa menor a $0.60 \quad(\alpha=0.37)$. Al realizar los análisis de segundo orden con las 15 escalas se encuentran que emergen tres factores a los que llamaron afrontamiento centrado en el problema, centrado en la emoción y evitativo. Aparentemente, estos resultados fueron similares a lo reportado por Carver et al. (1989) en su estructura tentativa de 4 factores. Sin embargo, en el estudio de Ingledew et al. (2013) la distribución de las estrategias presenta una diferencia. La misma consiste en que el estilo de afrontamiento cognitivo y el estilo orientado a la tarea aparecen fusionados en un factor llamado centrado en el problema.

También existen reportes psicométricos del funcionamiento de la versión de 60 ítems en España. Crespo y Cruzado (1997) analizaron las respuestas al COPE en 401 estudiantes universitarios, si bien los autores encuentran varias similitudes con la estructura de 15 factores planteada por Carver y sus colaboradores, del análisis factorial de segundo orden emergen 6 factores que representarían 6 estilos de afrontamiento: centrado en el problema (conductual y cognitivo), evitación (conductual y cognitiva), centrado en las emociones y uso de alcohol y drogas. Estos autores sugieren discutir los resultados en términos de dimensiones: aproximación-evitación, problema-emoción y conductual-cognitivo.

En Croacia, Hudek-Knežević, Kardum y Vukmirović (1999) analizaron el inventario en una versión independiente de 64 ítems en una muestra de 403 estudiantes universitarios. Estos autores prueban 4 modelos mediante análisis factoriales confirmatorios, brindando mayor apoyo a un modelo similar al de Ingledew et al. 
(1996) de 3 factores, a los que llaman afrontamiento orientado al problema, afrontamiento orientado a la emoción y desentendimiento, cabe señalar que en estos últimos análisis no incorporan las escalas humor, uso de drogas ni acudir a la religión. Por otro lado, se hallan índices de consistencia interna entre 0.52 y 0.87 para las estrategias y entre 0.82 a 0.91 para los estilos; además reportan índices de confiabilidad test-retest entre 0.43 a 0.72 en un intervalo de dos meses en 150 alumnos.

En Estonia, Kallasmaa y Pulver (2000) decidieron evaluar las propiedades psicométricas y estructura interna del COPE en su versión de 60 ítems, el estudio se llevó a cabo con 515 estudiantes universitarios. Los resultados muestran índices de consistencia interna entre 0.49 a 0.93 , y una confiabilidad test-retest, obtenida sobre 33 alumnos, entre 0.32 a 0.63 en un intervalo de 27 meses. La estructura factorial que aparece a través de un análisis de componentes principales con rotación varimax arroja una fórmula final de 3 factores que explican el $42 \%$ de la varianza, estos factores, nuevamente, son similares más no iguales a los planteados por Carver et al (1992) e Ingledew et al. (1996).

Finalmente, en Rumania existe una versión traducida y validada del COPE, esta fue realizada por Craşovan y Sava (2013) con 1009 sujetos adultos. Estos autores realizan un análisis factorial confirmatorio con el fin de poner a prueba seis modelos basados en la literatura científica existente, tampoco incorporan en esos análisis las escalas humor, uso de drogas ni acudir a la religión. Sus resultados brindan mayor apoyo a un modelo de 4 factores correlacionados, esos factores serían los mismos que los hallados por Carver et al (1989) y los llaman afrontamiento centrado en el problema, afrontamiento centrado en la emoción, afrontamiento centrado en el soporte social y afrontamiento evitativo. Además, calculan los índices de consistencia interna, hallando que para las estrategias son entre 0.48 a 0.92 y para las escalas entre 0.72 a 0.84 .

\section{Investigaciones en el Perú}

En el Perú, el COPE de 52 ítems fue adaptado por Casuso (1996) en una muestra de 817 universitarios. En ese estudio se encontró para las estrategias índices alfa de Cronbach que oscilan entre 0.40 y 0.86 , siendo las escalas con confiabilidad más bajas desentendimiento mental $(\alpha=0.48)$ y negación $(\alpha=0.40)$. A nivel de la validez, el análisis factorial exploratorio con rotación varimax arrojó 13 escalas con valores eigen mayores a 1 y adecuadas cargas factoriales, aunque la estructura factorial no coincidió totalmente con la original. Por ello, la investigadora sugiere una reorganización de la prueba, recomendación que no se sostiene en subsiguientes investigaciones.

Las siguientes investigaciones en el Perú que usan la adaptación de Casuso mantienen para los estilos la estructura teórica más no la empíricamente planteada por Carver et al (1989). Así se organizan las estrategias en términos de tres estilos: centrado en el problema (incluye afrontamiento activo, planificación, supresión de actividades en competencia, restricción del afrontamiento y búsqueda de apoyo social por razones instrumentales), centrado en la emoción (búsqueda de apoyo social por razones emocionales, reinterpretación positiva $y$ crecimiento, aceptación, acudir a la religión, negación, y enfocar/liberar emociones) y centrado en otros aspectos (enfocar y liberar emociones, desentendimiento conductual y desentendimiento mental).

Del análisis realizado, se puede concluir que el COPE se muestra como una evaluación finamente diferenciada de las diversas estrategias de afrontamiento, y la mayoría de los estilos presentan consistencia a lo largo de las investigaciones. Sin embargo, aún subsisten algunas dificultades por resolver respecto de su estructura, entre ellas se encuentran el funcionamiento de algunas escalas que estudios a nivel mundial y en el Perú reportan consistencias internas relativamente bajas, menores a 0.60. Entre esas escalas se hallan desentendimiento mental (Alcalde, 1998; Carver et al, 1989; Cassaretto, 2009; Chau, 1999; Chau, 2004; Kallasmaa \& Pulver, 2000); restricción del afrontamiento (Alcalde, 1998; Cassaretto, 2009; Casuso, 1996; Chau, 1999, 2004; Ficková, 2005; Kallasmaa \& Pulver, 2000), afrontamiento activo (Alcalde, 1998; Ficková, 2005) y negación (Alcalde, 1998; Casuso, 1996; Ficková, 2005). 
Además, en los análisis factoriales exploratorios de primer orden suelen emerger entre 10 a 13 factores iniciales debido a que de forma consistente ciertas escalas se agrupan bajo un solo factor (afrontamiento activo y planificación por un lado; búsqueda de soporte por razones instrumental y búsqueda de soporte por razones emocionales por otro).

Asimismo, en los análisis factoriales de segundo orden suelen emerger desde tres hasta cinco factores finales, los cuales pueden explicar de manera adecuada la varianza en los datos (Chengsun et al, 2002; Crespo \& Cruzado, 1997; Ficková, 2005; Kallasmaa \& Pulver, 2000; Lyne \& Roger, 2000). La evidencia parece indicar que los factores que mejor se replican corresponden la dimensión social/emocional (las dos escalas de búsqueda de soporte y liberación de emociones), seguido de la dimensión orientada a la evitación/desconexión (desentendimiento mental, desentendimiento conductual y negación).

Tomando en cuenta el uso extendido del COPE en su versión de 52 ítems dentro del contexto peruano e internacional, se hace necesario brindar un mayor fundamento sobre su funcionamiento psicométrico. Especialmente con la versión de 60 ítems, que presenta nuevas escalas de interés teórico y empírico para el estudio del afrontamiento dentro del campo de la salud.

Por ello, el propósito de este estudio es analizar las propiedades psicométricas del Inventario de Estimación del Afrontamiento (COPE) en un grupo de estudiantes universitarios de Lima, con el fin de analizar si se replican sus 15 escalas y si es posible, la estructura de estilos sugerida por distintos investigadores.

\section{Método}

\section{Participantes}

El presente estudio contó con la participación de 300 estudiantes de ambos sexos, de una universidad privada de Lima, Perú. Sus edades oscilaban entre los 16 y 25 años $(M=18,27$ y D.E. $=1,39$ años $)$. El $50,7 \%(\mathrm{n}=152)$ de los participantes fueron varones y el 49,3\% ( $n=148)$, mujeres. Los participantes eran estudiantes de primeros años de las facultades de letras $(53,3 \%$, $\mathrm{n}=160) \quad y \quad$ ciencias $\quad(46,7 \% . \quad \mathrm{n}=140) . \quad$ Los estudiantes aceptaron participar voluntariamente a través de un consentimiento informado escrito.

\section{Medición}

COPE es un inventario multidimensional que evalúa las diferencias individuales en las respuestas de afrontamiento, fue desarrollado en 1989 por Carver, Scheier y Weintraub. Este inventario está conformado por 60 ítems, que se califican en un formato Likert que va del 1 al 4, en los cuales el puntaje 1 equivale a casi nunca lo hago, 2 a veces hago esto, 3 usualmente hago esto y 4 hago esto con mucha frecuencia.

Como se señalara precedentemente en Latinoamérica, existe una versión traducida al español realizada por Casuso (1996), que incluye 52 ítems y otra versión de Calvete de 60 ítems, que incluye los 52 ítems originales, bastante similares en su traducción a los de Casuso, y 8 ítems adicionales que evalúan las dos nuevas escalas agregadas por los autores. Esta es la versión utilizada en este estudio, aunque se realizaron ligeros cambios en 10 ítems para mejor el fraseo de los mismos.

\section{Procedimiento}

Esta investigación se inició con estudio piloto con 100 estudiantes universitarios para evaluar la pertinencia lingüística de la prueba, determinándose que se utilizaría 46 ítems de la traducción de Calvete, 9 ítems de Casuso y 5 ítems nuevos, basados en un fraseo más comprensible para la población de estudio. La versión final de la prueba fue administrada, en una segunda fase, en diferentes aulas de los primeros años de carrera dentro de una universidad privada de Lima.

Los análisis de los resultados consistieron en estudiar primero el comportamiento de las estrategias de afrontamiento planteadas por el COPE. Para ello, se realizó un análisis factorial exploratorio con rotación oblicua, con el fin de analizar la estructura factorial del instrumento y la ubicación de los ítems en la misma. Además, se determinaron las correlaciones de cada uno de los ítems con su respectiva escala y la confiabilidad de cada uno de las estrategias mediante el coeficiente alfa de Cronbach. 
Posteriormente, se realizaron tanto un análisis factorial exploratorio como análisis confirmatorios para determinar si se podrían generar un reagrupamiento de las escalas en términos de grandes estilos de afrontamiento. Siguiendo las recomendaciones de autores como el propio Carver et al (1989), Crasovan y Sava (2013), Hudek-Knežević et al., (1999) e Ingledew et al. (1996) se excluyeron de los análisis las escalas humor, uso de drogas y acudir a la religión. Se siguieron los criterios de evaluación de la bondad de ajuste de cada modelo utilizados por los estudios con análisis factoriales confirmatorios con el COPE llevados a cabo por Hudek-Knežević et al. (1999) y Crașovan y Sava (2013). Se concluye presentando los indicadores de confiabilidad para los estilos hallados en los modelos.

\section{Resultados}

Para evaluar la validez de la prueba, se realizó un análisis factorial exploratorio, tal como el desarrollado por Carver y colaboradores en 1989. Se utilizó un análisis con rotación oblicua y extracción de componentes principales. Mediante estas técnicas se comprobó la pertinencia del uso del mismo ya que se alcanzaron valores adecuados $\quad\left[\mathrm{KMO}=0.798, p=0.01 ; x^{2}=8529.48\right.$; $\mathrm{gl}=1770, p<0.01]$.

Los resultados arrojan una solución que explica el 65,15\% de varianza para 15 factores. En esta estructura se observa que las estrategias afrontamiento activo y planificación forman un solo factor, y las estrategias buscar soporte social instrumental y buscar soporte social emocional forman otro factor. También se halla que cinco ítems (C25, C15, C1, C59 y C6) no presentan una adecuada carga factorial ya que no logran ubicarse en el factor al que teóricamente pertenecen o no cubren los criterios establecidos por Carver y colaboradores (1989) de tener una carga factorial superior al 0.30 .

La fusión de las escalas encontrada se entiende si uno analiza la tabla de correlaciones (Tabla 1) entre las escalas del COPE, en las que se halla que las escalas afrontamiento activo y planificación, así como la búsqueda de soporte instrumental como búsqueda de soporte emocional presentan correlaciones significativas y altas entre ellas.

Tabla 1. Correlación entre las escalas del COPE disposicional

\begin{tabular}{|c|c|c|c|c|c|c|c|c|c|c|c|c|c|c|}
\hline 1 & 2 & 3 & 4 & 5 & 6 & 7 & 8 & 9 & 10 & 11 & 12 & 13 & 14 & 15 \\
\hline 1 & $.62 * *$ & $.31 * *$ & $.40 * *$ & $.23 * *$ & $.12 *$ & $.47 * *$ & .10 & .11 & -.01 & $.19 * *$ & .01 & -.01 & .04 & $-.16 * *$ \\
\hline 2 & & $.37 * *$ & $.36^{* *}$ & $.23 * *$ & $.18^{* *}$ & $.51^{* *}$ & $.16^{* *}$ & $.14 *$ & $-.17 * *$ & .10 & -.03 & -.05 & -.02 & $-.33 * *$ \\
\hline 3 & & & $.28^{* *}$ & $.16^{* *}$ & .10 & $.36^{* *}$ & .10 & .04 & .03 & $.25^{* *}$ & $-.24 * *$ & -.04 & .11 & -.01 \\
\hline 4 & & & & $.23 * *$ & $.18^{* *}$ & $.24^{* *}$ & .11 & $.12 *$ & $.21^{* *}$ & $.20^{* *}$ & $.19^{* *}$ & -.01 & $.121 *$ & -.02 \\
\hline 5 & & & & & $.68 * *$ & $.31^{* *}$ & .08 & $.16 * *$ & $.20^{*}$ & .07 & $.25^{* *}$ & -.07 & .09 & -.03 \\
\hline 6 & & & & & & $.25^{* *}$ & .08 & $.20 * *$ & .01 & .03 & $.35^{* *}$ & -.05 & .06 & -.03 \\
\hline 7 & & & & & & & $.33 * *$ & $.14 *$ & -.04 & $.25^{* *}$ & -.07 & -.10 & .00 & $-.24 * *$ \\
\hline 8 & & & & & & & & -.10 & $.13^{*}$ & $.25^{* *}$ & .07 & $.17 * *$ & $.11 *$ & .09 \\
\hline 9 & & & & & & & & & $.17 * *$ & -.03 & $.16^{* * *}$ & $-.20 * *$ & $.17 * *$ & -.07 \\
\hline 10 & & & & & & & & & & -.04 & $.24 * *$ & .07 & $.35^{* *}$ & $.38 * *$ \\
\hline 11 & & & & & & & & & & & .01 & .11 & $.21 * *$ & $.16^{* * *}$ \\
\hline 12 & & & & & & & & & & & & $.15^{*}$ & $.19 * *$ & $.23 * *$ \\
\hline 13 & & & & & & & & & & & & & $.16^{* *}$ & $.19 * *$ \\
\hline 14 & & & & & & & & & & & & & & $.25 * *$ \\
\hline 15 & & & & & & & & & & & & & & 1 \\
\hline
\end{tabular}

(1. Activo, 2. Planificación, 3. Contención del afrontamiento, 4. Supresión de actividades competentes, 5. Búsqueda de soporte por motivos instrumentales, 6 . Búsqueda de soporte por motivos emocionales, 7. Reinterpretación positiva y crecimiento, 8 . Uso del humor, 9. Acudir a la religión, 10. Negación, 11. Aceptación, 12. Liberación de emociones, 13. Uso de drogas y alcohol, 14. Desentendimiento mental, 15. Desentendimiento conductual). $* p<0.05, * * p<0.01$ 
Tabla 2. Indicadores de consistencia interna del COPE

\begin{tabular}{lcc}
\hline \multicolumn{1}{c}{ Estrategias } & Alfas & $\begin{array}{c}\text { Rango de las } \\
\text { correlaciones } \\
\text { ítem - test }\end{array}$ \\
\hline Afrontamiento activo & .54 & $.14-.46$ \\
Planificación & .82 & $.58-.74$ \\
Restricción del afrontamiento & .55 & $.29-.43$ \\
Supresión de actividades en competencia & .62 & $.24-.53$ \\
Soporte Instrumental & .80 & $.54-.65$ \\
Soporte Emocional & .85 & $.54-.76$ \\
Reinterpretación & .66 & $.29-.48$ \\
Humor & .91 & $.69-.89$ \\
Religión & .88 & $.72-.81$ \\
Negación & .73 & $.40-.64$ \\
Aceptación & .68 & $.42-.51$ \\
Enfocar y liberar emociones & .75 & $.39-.66$ \\
Drogas & .89 & $.62-.81$ \\
Desentendimiento Mental & .53 & $.29-.35$ \\
Desentendimiento Conductual & .73 & $.39-.67$ \\
\hline
\end{tabular}

Se realizó un análisis de la confiabilidad de cada una de las estrategias y las correlaciones de los ítems con sus respectivas escalas. Se puede encontrar que la totalidad de las escalas superan el punto recomendado por Hernández Sampieri, Fernández Collado y Baptista Lucio (2010) para una consistencia media, pero cinco escalas no superan el punto de 0.7 recomendado como óptimo. Además, todos los ítems presenta correlaciones ítem-test positivas; solamente el ítem C25 ("Tomo medidas adicionales para intentar librarme del problema") presenta puntajes inferiores al 0.20 recomendado.

A fin de explorar la posibilidad de una estructura superior para las escalas, se procedió a realiza un análisis factorial exploratorio del cual emergió una estructura de 3 factores que explican el $55.92 \%$ de la varianza de la prueba $\left(\mathrm{KMO}=0,69, x^{2}=958,14 ; \mathrm{gl}=66 ; p<0.01\right)$.

$\mathrm{Al}$ factor número 1 lo conforman las estrategias que implican los esfuerzos cognitivos y conductuales más activos para afrontar al estresor. Al segundo factor lo conforman las estrategias más centradas en diversas formas de evitación del estresor. Al tercer factor lo conforman las escalas centradas en la búsqueda de contacto con otros. Todas las escalas superan el punto 0.4 de saturación dentro de su respectivo factor. Existe correlaciones pequeñas entre el primer factor y el tercero $(r=.21)$ y entre el segundo y el tercer factor $(r=.17)$. Finalmente, se pusieron a prueba tres modelos de organización de las escalas del COPE. El Modelo A incluía una propuesta de 3 factores conformados por 4 escalas cada uno, tal como los organizaba teóricamente Carver y colaboradores en 1989 (estilos: centrado en el problema, en la emoción y otras escalas).

Tabla 3. Análisis factorial exploratorio de las escalas del COPE

\begin{tabular}{lccc}
\hline & 1 & 2 & 3 \\
\hline Activo & $\mathbf{. 7 5}$ & & \\
Planificación & $\mathbf{. 7 5}$ & & \\
Contención & $\mathbf{. 6 8}$ & & \\
Supresión & $\mathbf{. 5 7}$ & & \\
Búsqueda soporte & .31 & & $\mathbf{. . 8 1}$ \\
$\begin{array}{l}\text { instrumental } \\
\text { Búsqueda soporte }\end{array}$ & & & \\
emocional & & & $\mathbf{. . 8 6}$ \\
Reinterpretación & $\mathbf{. 7 1}$ & & \\
Aceptación & $\mathbf{. 4 6}$ & .35 & \\
Negación & & $\mathbf{. 7 0}$ & \\
Liberación de emociones & & .37 & $\mathbf{- . 6 3}$ \\
$\begin{array}{l}\text { Desentendimiento mental } \\
\text { Desentendimiento }\end{array}$ & & $\mathbf{. 6 7}$ & \\
conductual & & $\mathbf{. 7 4}$ & \\
Porcentaje de varianza & $25.57 \%$ & $17.69 \%$ & $12.66 \%$ \\
\hline
\end{tabular}


El Modelo $B$ plantea 4 factores organizados por tres escalas cada uno (estilos: orientado a la tarea, a lo cognitivo, a lo social-emocional y a la evitación), tal como lo hallado empíricamente por estos mismos autores. Finalmente en el Modelo $C$, que se organiza en 3 factores tal como lo hallado por Ingledew et al (1996), los estilos incluirían al problema o enfrentamiento (6 escalas), socialemocional (3 escalas) y evitativo (3 escalas), estructura similar a la hallada por Casuso (1996).

Los resultados finales, citados en la Tabla 4, desestiman el Modelo A de tres factores ya que la casi totalidad de medidas de ajuste al modelo hipotetizado no se acercan a los requerimientos mínimos sugeridos, lo cual indica un mal ajuste de dicho modelo.

Respecto a los Modelos $B$ y $C$ existe cierta evidencia que los soporta a ambos, aunque algunos de sus indicadores no logran ser plenamente satisfactorios. Respecto al $\chi^{2}$, este se muestra en los dos casos como significativo, lo cual sería un indicador de que la matriz de covarianzas observada y estimada no se ajusta plenamente; sin embargo, este es un indicador dependiente del tamaño de la muestra. Por ello, se analizó el radio recomendado entre el $\chi^{2}$ y sus respectivos grados de libertad, al hallarse que éste era menor a 5, se considera que el indicador es aceptable (Hair, Anderson, Tatham, \& Black, 1998).

Respecto a los indicadores GFI, AGFI y RMSR de ambos modelos se halla que estos cumplen las recomendaciones de Coover, Penner y MacCallum (1990) y Marsh, Balla y McDonald (1988); es decir, GFI $\geq 0.85$; AGFI $\geq 0.80$ y RMSR $\leq 0.10$ (como se cita en Hudek-Knežević et al., 1999) lo que da un apoyo aceptable de la bondad de ajuste del modelo a los datos. Sin embargo, el indicador CFI tiene un valor de inferior a 0.95 recomendado como adecuado y a 0.85 como aceptable, y los indicadores RMSEA son mayores a 0.06 (Schreiber et al., 2006). Estos indicadores advierten de la posibilidad de ajuste menos satisfactoria de ambos modelos y sugieren que la evidencia hallada debe tomarse con prudencia. Al analizar la saturación de cada escala dentro de los factores en el Modelo B se encuentra que todas superan el punto 0.30. Existen correlaciones entre algunos de los factores obtenidos, siendo las más importantes entre el primer factor y el tercero $(r=0.30)$, entre el primero y el cuarto $(r=-0.23)$, entre el segundo y el tercero $(r=0.38)$. Llama la atención la alta correlación entre el primer y segundo factor $(r=0.80)$, que podría sugerir que ambos factores pueden ser tratados como uno solo, debido a ello se sugiere la utilización del Modelo $\mathrm{C}$, de tres factores.

La Figura 1 presenta la saturación de cada escala del Modelo $C$, en ella se observa que todas las escalas superan el punto 0.30, salvo la escala aceptación (0.28). Entre las correlaciones entre algunos de los factores obtenidos, las más importantes aparecen entre el primer factor y el segundo $(r=0.34)$.

Figura 1. Análisis factorial confirmatorio para el modelo $\mathrm{C}$ de 3 factores

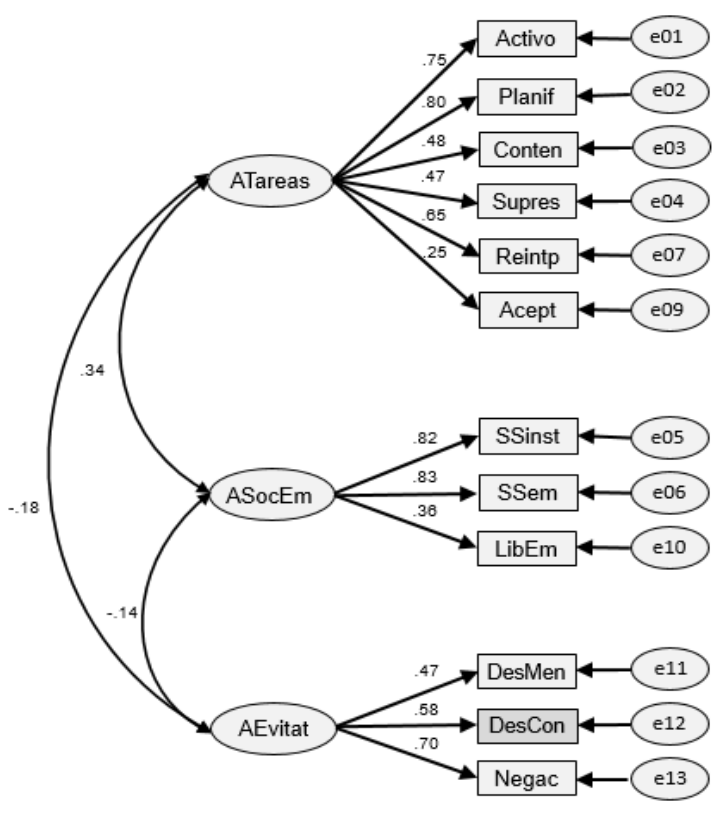

$\mathrm{Al}$ optar por este último modelo de tres factores se procedió a realizar los análisis de consistencia interna para los factores, hallándose índices alfa de 0.73 para el primer factor, de 0.59 para el segundo y 0.70 para el tercero. 
Tabla 4. Indicadores de bondad de ajuste para el COPE

\begin{tabular}{|c|c|c|c|c|c|c|}
\hline & $\chi^{2}$ & GFI & AGFI & RMSR & CFI & RMSEA \\
\hline $\begin{array}{c}\text { Modelo A: } 3 \\
\text { factores }\end{array}$ & $\begin{array}{c}1673.26 \\
(\mathrm{gl}=87) \\
p<0.01\end{array}$ & 0.8 & 0.73 & 0.05 & 0.53 & 0.14 \\
\hline $\begin{array}{l}\text { Modelo B: } 4 \\
\text { factores }\end{array}$ & $\begin{array}{c}235.84 \\
(\mathrm{gl}=48) \\
p<0.01\end{array}$ & 0.89 & 0.82 & 0.03 & 0.79 & 0.11 \\
\hline $\begin{array}{l}\text { Modelo C: } 3 \\
\text { factores }\end{array}$ & $\begin{array}{c}250.88 \\
(\mathrm{gl}=51) \\
p<0.01\end{array}$ & 0.89 & 0.82 & 0.03 & 0.78 & 0.11 \\
\hline
\end{tabular}

\section{Discusión}

Durante los últimos 40 años se viene trabajando exhaustivamente el concepto afrontamiento a nivel mundial; su relevancia radica en la posibilidad que nos ofrece de comprender mejor la forma en que los individuos manejan las situaciones adversas y cómo estas respuestas pueden afectar la salud de la persona (Barra, 2003; Taylor, 2007; Zeidner \& Endler, 1996).

Debido a su importancia, la evaluación adecuada del afrontamiento resulta necesaria. Desafortunadamente, son pocas las medidas que muestren de forma consistente indicadores de validez y confiabilidad. Sin embargo, entre las medidas más estudiadas a nivel mundial se encuentra el COPE, inventario creado por Carver, Scheier y Weintraub en 1989. Este es considerado una de las principales herramientas para medir las estrategias e identificar los estilos de afrontamiento (Clark et al., 1995; Ficková, 2005; Folkman \& Moskowitz, 2004; Kallasmaa \& Pulver, 2000; Lyne \& Roger, 2000; Schwarzer \& Schwarzer, 1996).

Este estudio, plantea revisar el funcionamiento psicométrico del COPE en su versión de 60 ítems dado que los estudios en el contexto peruano señalan solo las fortalezas y debilidades de la versión de 52 ítems (Alcalde, 1998; Cassaretto, 2009; Casuso, 1996; Chau, 1999, 2004; Rojas, 1997). Los resultados finales de esta investigación arrojan evidencia sólida respecto a la estructura y consistencia de las 15 estrategias de afrontamiento de la prueba. Se encuentra que emergen 13 factores claramente diferenciados, donde un factor está conformado por las escalas afrontamiento activo y planificación, mientras otro factor por las escalas búsqueda de soporte instrumental como búsqueda de soporte emocional.

La fusión de estas escalas es consistente con lo hallado en la investigación original de Carver y sus colaboradores (1989), pero también con otras investigaciones (Cassaretto, 2009; Clark et al., 1995; Chau, 2004). Dado las altas correlaciones entre cada par de escalas, ello nos lleva a considerar que ambas escalas en realidad miden disposiciones comunes. Así, aquellos que tienden a responder al estrés siendo activos, necesitarán ser capaces de planificar sus acciones previamente para ser más eficaces y sostener este tipo de afrontamiento en situaciones futuras. Además, aquellos que tienen facilidad para recurrir a otras personas para enfrentar situaciones estresantes, pueden ver en la ayuda recibida no solo alternativa para solucionar sus problemas sino una fuente de alivio ante las circunstancias. Sin embargo, Carver y sus colaboradores (1989) advierten que es posible que también cada estrategia pueda mostrar, en determinadas situaciones, tendencias distintas $\mathrm{y}$, por tanto, consideran relevante mantener una medición diferenciada de ambos aspectos.

Este estudio muestra diversas escalas que reflejan una estructura sólida y consistente. Además, en el mismo se encuentra que, con los cambios en el fraseo de ítems, la mayoría de las 
escalas tienen mejor funcionamiento a lo obtenido en investigaciones previas realizadas con la versión traducida de Casuso del 1996 (Cassaretto 2009; Chau, 1999, 2004; Rojas, 1997).

Llama la atención que nuevamente la escala menos consistente sea Desentendimiento mental, escala que evalúa cómo la persona utiliza una amplia variedad de actividades con el fin de distraerse y evitar la evaluación del problema. Recordando que otros estudios encuentran índice alfa bastante bajos en la misma (Alcalde, 1998; Carver et al., 1989; Casuso, 1996; Chau, 2004; Cassaretto, 2009; Craşovan \& Sava, 2013; Ficková, 2005; Ingledew, et al., 1996; Kallasmaa $\&$ Pulver, 2000) y, pese que ha habido intentos de fortalecer la escala con 7 ítems (Hudek-Knežević, et al., 1999), su consistencia interna sigue siendo relativamente baja.

Ello nos lleva a cuestionar los ítems de la escala Desentendimiento Mental, ya que si bien estos pueden referir cierta forma de distracción mental (por ejemplo "Sueño despierto con otras cosas diferentes" o "Duermo más de lo habitual"), básicamente describen conductas y no la función que estas cumplen, por lo que no son suficientemente explicitas como asegurar que evalúan las respuestas que se realizan con fines más evitativos.

Ello también nos lleva a discutir el delicado equilibrio que se establece entre afrontamiento disposicional y situacional, ya que si bien ambas dimensiones del afrontamiento están íntimamente relacionadas (Carver et al, 1989; FernándezAbascal, 1997; Krohne 1996), en algunas escalas es más clara la relación de las respuestas del afrontamiento con tendencias más disposicionales como la que corresponden a la personalidad, mientras que en otras no existe dicho vínculo (Carver y Connor-Smith, 2010; Cassaretto, 2011; Connor-Smith \& Flachsbart, 2007; Contreras, Espinoza, \& Esguerra, 2009; Snyder, 1999). En el COPE la naturaleza de los ítems de varias escalas reflejan con mayor claridad tendencias relativamente estables de afrontamiento mientras que otras respuestas se encuentran más ligadas a variables situaciones y de desarrollo. Por tanto, la motivación por la que se realizan las conductas reportadas no es fácilmente evaluada y en consecuencia por ejemplo, no se puede diferenciar si las acciones realizadas son con fines evitativos o con la intención de regular afectos.

Cabe señalar que se encontraron en este estudio 3 ítems que mostraron problemas tanto en la adecuación a la estructura factorial planteada por los autores, como por su pobre capacidad discriminativa respecto de la consistencia de la escala. Los ítems fueron C25 (tomo medidas adicionales para intentar librarme del problema) del área activo, $\mathrm{C} 15$ (evito distraerme con otros pensamientos o actividades) del área supresión y $\mathrm{C} 1$ (intento desarrollarme como persona como resultado de la experiencia) del área reinterpretación positiva.

La intención de esta investigación era que con traducciones más adecuadas de los ítems problemáticos reportados de la versión de Casuso (1996) se pudiera obtener una versión más fina del COPE en español. Sin embargo, de los 8 ítems reemplazados solo cinco tuvieron un mejor funcionamiento y tres de ellos $(\mathrm{C} 25, \mathrm{C} 15 \mathrm{y} \mathrm{C} 1)$ no mostraron mejora significativa en comparación a la versión anterior. Una posible explicación es que la fuente de dificultades con estos ítems no radica en la calidad de la traducción, sino en que presentan serios problemas para representar conductas propias de algunas estrategias de afrontamiento. Se observa que en los 3 ítems aparecen problemas para graficar claramente la intención por la que se realiza la conducta mencionada, por tanto el enfoque con el que se realiza esos afrontamientos no quedan claros. Se sugiere observar el comportamiento psicométrico de estos ítems en otras muestras con el fin de analizar su posible reemplazo en caso que se encuentre resultados similares.

A pesar de ello, encontramos que el COPE con sus 15 escalas demuestra ser una medida sólida para evaluar de las estrategias de afrontamiento. Sin embargo, uno de los aspectos de interés de esta investigación era analizar la posibilidad de organizar estas escalas en una estructura jerarquiza superior que refleje tendencias más estables y con un orden teórico de base. Son muchos los estudios que han intentado realizar dicha organización; sin embargo, no existe consenso sobre las formas de agrupar dichas tendencias del afrontamiento (Carver \& 
Connor-Smith, 2010; Folkman \& Moskowitz, 2004; Taylor, 2007).

En este estudio se intentó poner a prueba tres modelos de organización del COPE. Estos modelos son similares entre si y rescatan la consistencia de lo hallado en las diversas investigaciones que plantean como mínimo tres factores que pueden explicar de manera adecuada la varianza en los datos (Chengsun et al, 2002; Crasovan \& Sava, 2013; Crespo \& Cruzado, 1997; Ficková, 2005; Hudek-Knežević et al., 1999; Ingledew et al., 1996; Kallasmaa \& Pulver, 2000; Lyne \& Roger, 2000).

En varios de estudios se excluían de los análisis de segundo orden las escalas humor, uso de drogas y acudir a la religión, lo cual se fundamenta en que dichas escalas no logran consistentemente localizarse dentro de algún factor superior. Nuevamente, consideramos que si bien estas 3 estrategias describen claramente las conductas que realizan los individuos para afrontar el estrés no son claras la función que cumplen pudiendo responder a diversas estrategias $\mathrm{y}$ estilos de afrontamiento. Por ejemplo, las personas pueden acudir a la religión tanto para obtener alivio emocional, recibir soporte como obtener un medio que los distraiga de las preocupaciones.

Adicionalmente, esto podría reflejar que estas respuestas podrían estar afectadas por determinados contextos socioculturales. Por ejemplo, el uso del humor se ha agrupado en el factor "Evitación" (en versiones Italiana y Croatas; Huden-Knezevic, Kardum, \& Vukmirovic, 1999), en el factor “Tarea" (en la versión de Estonia; Kallasmaa \& Pulver, 2000), o en ningún factor de manera significativa (Carver et al, 1992). Ello puede explicarse ya que el uso del humor como estrategia única de afrontamiento ha sido cuestionado en la última década por diversos autores, que consideran que puede expresarse en varios estilos claramente diferenciados (Cann \& Etzel, 2008; Kerkkanen, Kuiper \& Martin, 2004; Kuiper, Grimshaw, Leite, \& Lirsh, 2004; Martin, 2007; Martin, PuhlikDoris, Larsen, Gray, \& Weir, 2003). En ese sentido, también sugerimos sostener este procedimiento de análisis en próximas investigaciones y excluir estas 3 escalas de los análisis de segundo orden.

Respecto a los modelos puestos a prueba en esta investigación, se encuentra que los datos claramente no se adecuan al modelo más cercano a la estructura teóricamente planteada por Carver y sus colaboradores (1989) que agrupa las estrategias en estilos centrados en el problema, centrados en la emoción y otros estilos. Esta estructura, si bien usada en diversas investigaciones por su interés práctico, no ha logrado ser reproducida en ninguna otra investigación; por ello, se sugiere desestimar su uso.

Respecto a los otros dos modelos, son similares respecto a dos de los factores presentados. Por un lado, la literatura científica deja clara la tendencia a organizar en un solo factor las dos escalas de búsqueda de apoyo social y la escala enfocar y liberar emociones, que corresponderían a una tendencia complementaria social/emocional del afrontamiento, dicho factor se reproduce claramente en nuestro análisis factorial exploratorio. Al respecto, Folkman y Moskowitz (2004) critican las visiones del afrontamiento individualista y sugieren una posible organización en tendencias de afrontamiento individual vs social; el afrontamiento social incorporaría elementos de manejo de emociones en contextos sociales ya que su expresión se encuentra ligada con aspectos relacionales.

Un segundo factor, consistentemente hallado en la literatura científica, integra las escalas desentendimiento mental, desentendimiento conductual y negación en otro factor que refleja la tendencia a la evitación o desconexión como formas de afrontamiento. Nuevamente se encuentra en el análisis factorial exploratorio que dicho factor se agrupa de forma robusta y nos refiere con claridad las respuestas evasivas en el afrontamiento que se encuentran teorizada por autores como Carver y Connor-Smith (2010).

Sin embargo, el resto de escala puede presentarse en uno o dos factores, es por ello que los análisis factoriales confirmatorios buscaban aclarar cuál de los modelos presentan una mejor representación de la estructura del COPE. Los resultados no son concluyentes, si bien existe 
cierto apoyo para estos dos últimos modelos en tanto cumple los criterios usados para evaluar la bondad de ajuste en la validación del COPE en una muestra Croata (Hudek-Knežević et al., 1999). En próximas investigaciones que usen técnicas de análisis factoriales confirmatorios se recomiendan adicionar criterios más exigentes (Schreiber et al., 2006).

Ello nos lleva a afirmar que la evidencia aún es insuficiente como para afirmar que alguno de estos modelos debe ser usado definitivamente. Mas cuando se encuentra que la escala aceptación presenta una saturación relativamente baja dentro del factor con el que se la agrupa. Sin embargo, se plantea usar de forma prudente el modelo de 3 factores que integra en los estilos orientado a lo social/emocional (búsqueda de apoyo social por razones instrumentales, búsqueda de apoyo social por razones emocionales y enfocar y liberar emociones), a la evitación (negación, desentendimiento conductual y desentendimiento mental) y a la tarea/aproximación (afrontamiento activo, planificación, supresión de actividades en competencia, restricción del afrontamiento, reinterpretación positiva $\mathrm{y}$ crecimiento, aceptación).

Dicho apoyo al modelo de 3 factores se fundamente en los resultados del análisis factorial exploratorio como en la evidencia obtenida desde los estudios de Hudek-Knežević, et al., (1999); Ingledew et al., (1996) y Kallasmaa y Pulver (2000). Sin embargo, nuevas investigaciones con esta versión podrán dar evidencia más concluyente sobre la pertinencia y solidez de esta última propuesta de organización de la prueba.

La medición del afrontamiento no es una tarea fácil pero es necesaria, por ello, debemos recordar que las técnicas de medición cuantitativa presentan sus limitaciones, entre ellas que se recurre al recuerdo para responder a los enunciados de las pruebas, la extensa longitud de los instrumentos y que estos han sido adaptados en la mayoría de casos en grupos de estudiantes universitarios. Por tales consideraciones, la medición fina del afrontamiento requiere validar los instrumentos ya conocidos, así como crear aquellos que sean más pertinentes a las características de desarrollo, contexto social y cultural de aquellos a quienes se necesita evaluar.
En esa línea, cabe recordar que el afrontamiento puede ser visto tanto en sentido disposicional o genérico como también en sentido situacional o específico; varios autores consideran que esos enfoques son complementarios (Carver et al, 1989; Carver \& Connor-Smith, 2010; Fernandez-Abascal, 2002). Por ello, no se debería abandonar el estudio de instrumentos de afrontamiento específicos, especialmente aquellos que evalúan los modos de afrontamiento propios de nuestros contexto frente a circunstancias especial de vida como pueden ser la adaptación a una enfermedad crónica (Lazcano-Ortiz, SalazarGonzález, \& Gómez-Meza 2008; Soriano \& Monsalve, 2004; Torres, Pereira \& Montero, 2014), a las demandas académicas (Cabanach, Valle, Rodríguez, Piñero, \& Freire, 2010; Fernández, Contini, Ongarato, Saavedra, \& de la Iglesia, 2009) o que toman en consideración la etapa de vida de la persona (Canessa, 2002; Richaud De Minzi \& Iglesias, 2013) entre otros.

\section{Referencias}

Alcalde, M. (1998). Nivel de Autoeficacia percibida y estilos de afrontamiento en estudiantes universitarios de Lima. Tesis para optar el título de Licenciada en Psicología, con mención en psicología clínica, no publicada, Pontificia Universidad Católica del Perú, Lima.

Barra, E. (2003). Psicología de la salud. Santiago de Chile: Editorial Mediterráneo Ltda.

Cabanach, R., Valle, A., Rodríguez, S., Piñero, I., $\&$ Freire, C. (2010). Escala de Afrontamiento del Estrés Académico (A-CEA). Revista Iberoamericana de Psicología y Salud, 1(1).

Canessa, B. (2002). Adaptación psicométrica de las Escalas de Afrontamiento para Adolescentes de Frydenberg y Lewis en un grupo de escolares de Lima metropolitana, Persona 5,191-233.

Cann, A., \& Etzel, K. (2008). Remembering and anticipating stressors: Positive personality mediates the relationship with sense of humor. Humor: International Journal of Humor Research. 21(2), 157-178.

Carver, C., Scheier, M., \& Weintraub, J. K. (1989). Assessing Coping Strategies: A 
theoretically based approach. Journal of Personality and Social Psychology, 56(2), 267-283.

Carver, C., \& Connor-Smith, J. (2010). Personality and coping. Annual review of Psychology, 61,679-704.

Cassaretto, M. (2009). Relación entre las cinco grandes dimensiones de la personalidad y el afrontamiento en pre-estudiantes universitarios de Lima Metropolitana. Tesis de maestría no publicada. Universidad Nacional Mayor de San Marcos, Lima, Perú.

Cassaretto, M. (2011). Relaciones entre la personalidad y el afrontamiento en estudiantes preuniversitarios. Revista Vanguardia Psicológica, 1(2), 202-225.

Cassaretto, M., Chau, C., Oblitas, H., \& Valdés, N. (2003). Estrés y afrontamiento en estudiantes de psicología. Revista de Psicología de la Pontificia Universidad Católica del Perú, 21(2), 363-392.

Cassaretto, M., \& Paredes, M. (2006). Afrontamiento a la enfermedad crónica: Estudio en pacientes con insuficiencia renal crónica terminal. Revista de Psicología, 24(1), 109-142.

Casuso, L. (1996). Adaptación de la prueba COPE sobre estilos de Afrontamiento en un grupo de estudiantes universitarios de Lima. Tesis para optar el título de Licenciada en Psicología con mención en psicología clínica no publicada. Pontificia Universidad Católica del Perú, Lima.

Chau, C. (1999). Consumo de bebidas alcohólicas en estudiantes universitarios: Motivaciones y estilos de afrontamiento. Persona. Revista de la Facultad de Psicología. Universidad de Lima, 2, 121-161

Chau, C. (2004). Determinants of alcohol use among university students: The role of stress, doping and expectancies. Amsterdam: Katholieke Universiteit Leuven.

Chau, C., Morales, H., \& Wetzell, M. (2002). Estilos de afrontamiento y estatus performance en un grupo de pacientes oncológicos hospitalizados. Revista de Psicología de la PUCP, 20(1), 93-131.

Chengsun, S., Yan, D., Yubo, H., Guizhi, H., \& Xiaomei, Z. (2002). Preliminar development of the COPE Scale. Acta Psychologica Sinica, 34(4), 414-420.

Clark, K., Bormann, C. Cropanzano, R., \& James, K. (1995). Validation evidence for three coping measures. Journal of Personality Assessment, 65(3), 434-455.

Connor-Smith, I., \& Flachsbart, C. (2007). Relations between personality and coping: A meta-analysis. Journal of Personality and Social Psychology, 93(6), 1080-1107.

Contreras, F., Espinoza, J., \& Esguerra, G. (2009). Personalidad y afrontamiento en estudiantes universitarios. Universitas Pshychologica, 8(2), 311-322.

Cooper, C. (2013), (Ed.). From Stress to Wellbeing. Vol 2: Stress Management and Enhancing Well-being. New York: PalgraveMcMillan

Craşovan, D., \& Sava, F. (2013). Translation, adaptation, and validation on romanian population of COPE Questionnaire for Coping Mechanisms Analysis. Cognition, Brain \& Behavior. An Interdisciplinary Journal, 17(1), 61-76.

Crespo, M., \& Cruzado, J. (1997). La evaluación del afrontamiento: Adaptación Española del Cuestionario COPE con una muestra de estudiantes universitarios. Análisis y Modificación de Conducta, 23(92), 797-830.

Fernández-Abascal, E. (2002). Psicología general: Motivación y emoción. Madrid. Editorial centro de estudios Ramón Areces S.A.

Fernández, M., Contini, N., Ongarato, P., Saavedra, E., \& de la Iglesia, G. (2009). Estrategias de afrontamiento en estudiantes medios y universitarios. Revista Iberoamericana de Diagnóstico y Evaluación - e Avaliação Psicológica, 27(1), 63-84.

Ficková, E. (2005). Coping scales for adults: Psychometric relationships. Studia Psychologica, 47(4), 309-317.

Folkman, S., \& Moskowitz, J. (2004) Coping: Pitfalls and promise. Annual Review of Psychology, Vol 55, 745-74.

Hernández Sampieri, R., Fernández Collado, C., \& Baptista Lucio P. (2010). Metodología de Investigación. México D.F.: McGraw - Hill. 
Gastelumendi, C., \& Ore, B. (2013). Personalidad y afrontamiento en voluntarios peruanos de lucha contra la pobreza. Revista de Psicología, 31(1), 67-98.

Hair, J., Anderson, R., Tatham, R., \& Black, W. (1998). Multivariate data analysis $\left(5^{\text {th }}\right.$ ed.). New Jersey: Prentice-Hall.

Hudek-Knežević, J., Kardum, I., \& Vukmirović, Z. (1999). The Structure of coping styles: A comparative study of croatian sample. Europan Journal of Personality, 13, 149-161.

Ingledew, D. K., Hardy, L., Cooper, C.L., \& Jemal, H. (2013). Health behaviors reported as coping strategies: A factor analytical study. En C. Cooper (Ed.). From stress to wellbeing. Vol 2: Stress management and enhancing well-being. New York (pp. 62-84). London: Palgrave-McMillan Ed.

Kallasmaa, T., \& Pulver, A. (2000). The structure and properties of the Estonian COPE inventoy. Personality and Individual Differences, 29, 881-894.

Kerkkänen, P. Kuiper, N., \& Martin, R. (2004). Sense of humor, physical health, and wellbeing at work: A three-year longitudinal study of finnish police officers. Humor: International Journal of Humor Research Vol. 17(1-2), 21-35.

Krohne, H. W. (1996). Individual differences in coping. En M. Zeidner \& N.S. Endler (Eds.), Handbook of coping: Theory, research, applications, (pp. 381-409). Nueva York: John Wiley \& Sons.

Kuiper, N., Grimshaw, M., Leite, C., \& Lirsh, G. (2004). Humor is not always the best medicine: Specific components of sense of humor and psychological well-being. International Journal of Humor Research 17(1/2), 135-168.

Lazcano-Ortiz, M., Salazar-González, B., \& Gómez-Meza, M. (2008). Validación del instrumento: afrontamiento y proceso de adaptación de Roy en pacientes con diabetes mellitus tipo 2. Aquichán, 8(1), 116-125.

Levano, J. (2003). El patrón de conducta Tipo A y Tipo B y los estilos de afrontamiento al estrés en una muestra de personal militar. Tesis para obtener el grado de Magíster en Psicología no publicada. Lima: Universidad Nacional Mayor de San Marcos.

Lyne, K., \& Roger, D. (2000). A psychometric reassessment of the COPE questionnaire. Personality and Individual Differences, 29, 321-335.

Martin, R. (2007). The psychology of humor: An integrative approach. San Diego: Elsevier Inc.

Martin R., Puhlik-Doris P., Larsen W., Gray J., \& Weir, K. (2003) Individual differences in uses of humor and their relation to psychological well-being: Development of the Humor Styles Questionnaire. Journal of Research in Personality $N^{\circ} 37,48-75$.

Richaud de Minzi, M., \& Iglesias, M. (2013). Evaluación del afrontamiento en niños pequeños. Subjetividad y Procesos Cognitivos, 17 (1), 244-256.

Rojas, R. (1997). Ansiedad, cólera y estilos de afrontamiento en portadores de VIH. Tesis para obtener el grado de Magíster en Psicología no publicada. Lima: Pontificia Universidad Católica del Perú

Schreiber, J., Nora, A., Stage, F., Barlow, E., \& King, J. (2006). Reporting structural equation modeling and confirmatory factor analysis. A review. Journal of Educational Research, 99, 323-337.

Schwarzer, R., \& Schwarzer,, Ch. (1996). A critical survey of coping instruments. En M. Zeidner \& N.S. Endler (Eds.), Handbook of coping: Theory, research, applications, (pp. 107-132). Nueva York: John Wiley \& Sons.

Snyder, C. (1999). Coping: The psychology of what works. New York: Oxford University Press.

Solano, M. (2013). Relaciones entre el dolor por artritis reumatoide con el bienestar psicológico, afrontamiento y síntomas depresivos. Tesis de maestría. Pontifica Universidad Católica del Perú. Lima, Perú.

Soriano, J., \& Monsalve, V. (2004). Validación del cuestionario de afrontamiento al dolor crónico reducido (CAD-R). Revista de la Sociedad Española del Dolor, 11 (7), 407414. 
Taylor, S. (2007). Psicología de la Salud (6ta Edición). México D.F.: McGraw Hill Interamericana.

Torres, A., Pereira, A., \& Monteiro, S. (2014). Estudo de validade da versão portuguesa do Questionário de Formas de Lidar com o Cancro. Revista Iberoamericana de Diagnóstico y Evaluación - e Avaliação Psicológica, 38 (2), 199-217.

Zeidner, M., \& Endler, N. S. (Eds.). Handbook of coping: Theory, research, applications. Nueva York: John Wiley \& Sons. 